\title{
Christophe Sireix
}

avec la collaboration * de

Bernard Abaz

Alain Beyneix

Yves Marcadal

Alain Réginato

\section{Officines de potiers du Second Age du Fer dans le sud-ouest de la gaule : Organisation, structures de cuisson et productions}

\section{Résumé}

Un grand nombre de fours de potiers du Second Age du Fer a pu être recensé et observé dans le sud-ouest de la Gaule. Ces fours sont associés à des habitats groupés (de plaine ou de hauteur) établis le long des principaux axes fluviaux: la Garonne et la Dordogne. Ils appartiennent souvent à de véritables officines.

L'étude de l'organisation, de la conception et de la production de ces fours, permet de constater les nombreuses similitudes qui les caractérisent.

\begin{abstract}
A great deal of pottery kilns from the Second Iron Age have been inventored in the south-west Gaul. They are all connected to clustered habitat (located in plain or hight) along the main rivers: the Garonne and the Dordogne. They often belong to real production centers.
\end{abstract}

The study of their organisation, their conception and their production brings into light the many common points whitch caracterize all this kilns. 


\section{Fig. 1.}

Répartition des fours $d u$ Second Age du Fer en Aquitaine,

1. Sainte-Sainte-

Florence (33), Les

Grands Vignes ;

2. Mouliets-et-

Villemartin (33),

Lacoste ;

3. Lagruère (47),

Au Bourg ;

4. Aiguillon (47),

La Gravisse :

5. Agen (47),

L'Hermitage ;

6. SOS (47),

Peyroutet.

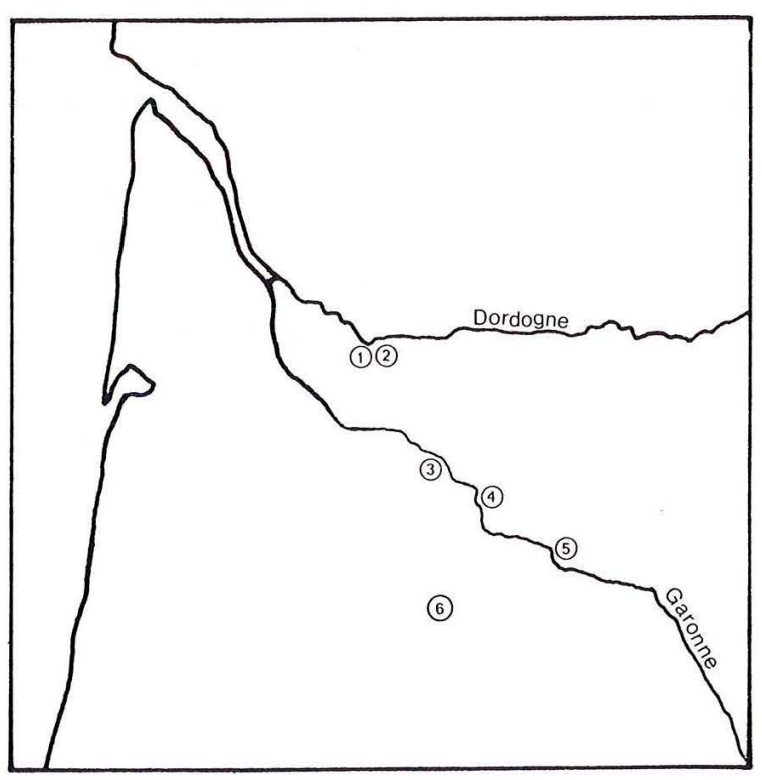

Le sud-ouest de la Gaule, ou plutôt l'Aquitaine au sens césarien du terme, réunit sur son territoire un nombre important de fours de potiers du second age du Fer. 37 fours y sont actuellement recensés, et si l'on agrandit quelque peu ce cadre géographique, on atteint une concentration surprenante avec un total de plus de 60 fours répartis entre la Méditerranée et l'Atlantique, le long de l'axe garonnais ou en proche périphérie.

\section{Répartition géographique}

Les 37 fours qui nous intéressent ici, se situent géographiquement de la manière suivante (fig. 1) :

- Le Lot-et-Garonne domine largement les autres départements.

Dix fours sont connus sur le site de Sos, aux lieux dits «Campet et Peyroutet» '. Cinq fours ont été exhaustivement fouillés sur le site de «La Gravisse» à Aiguillon ${ }^{2}$, quatre à Lagruère, au lieu-dit «Le Bourg» (un cinquième n'a été que simplement repéré ${ }^{3}$ ). Sur l'oppidum de 1' «Ermitage» à Agen ${ }^{4}$, deux fours de potiers ontété découverts en 1869 , puis publiés quelques années plus tard.

- En Gironde, onze fours sont connus sur le site de «Lacoste» à Mouliets-et-Villemartin ${ }^{5}$, deux sur le site des «Grands Vignes II» à Sainte-Florence ${ }^{6}$.
- Pour le Gers, nous signalerons simplement la présence de deux fours isolés dont la datation et la description ne sont pas suffisamment précises pour qu'ils soient pris en compte ; l'un a été découvert près de Lectoure ?, à proximité des puits funéraires de «Lamarque», l'autre dans un faubourg de la ville d'Auch ${ }^{8}$.

En dehors de ces deux derniers fours d'attribution chronologique un peu douteuse, nous avons donc recensé 35 fours répartis sur seulement 6 sites parmi lesquels 5 sont dotés de véritables ateliers de production, d'officines : il s'agit des sites d'Agen ${ }^{9}$, Mouliets-etVillemartin, Aiguillon, Lagruère et Sos. Tous les fours découverts sur ces sites sont datables de la phase finale du second age du Fer. Seuls les deux fours du site des «Grands-Vignes II» appartiennent à une phase plus ancienne (IVe siècle avant J.-C.), ils peuvent être considérés comme des petites unités de production isolées. Nous reviendrons sur ces derniers à la fin de cette étude.

Dans un premier temps, nous allons voir comment s'articulent ces fours par rapport aux habitats dont ils dépendent, décrire leur organisation au sein même des officines, déterminer leurs caractéristiques morphologiques et enfin définir les principaux traits de leur production.

1. Marcadal Y.,L'âge du Feren Agenais, thèse de IIIe cycle, dactylographiée, Bordeaux, 1971, p. 161-169 et Lapart J., Fours de potiers gaulois à Sos, Revue de l'Agenais, 2, 1982, p. 171-185.

2. Réginato A., Les Celtes, la Garonne et les pays aquitains, livret-guide de l'exposition du Musée des Beaux-Arts d'Agen, 1992, p. 78-79.

3. Abaz B., Les Celtes, la Garonne et les pays aquitains, livret-guide de l'exposition du Musée des Beaux-Arts d'Agen, 1992, p. 80-82.

4. Magen A., Notice sur deux fours de potiers de l'époque gallo-romaine, Recueil des travaux de la Société d'Agriculture, Lettres et Arts d'Agen, III, 1873, p. 55-67.

5. Sireix C., Officine de potiers et production céramique sur le site protohistorique de Lacoste à Mouliets-et-Villemartin (Gironde) Aquitania, VIII, 1990, p. 45-97.

6. Sireix C., Le site protohistorique des Grands-Vignes II à Sainte-Florence (Gironde), Aquitania, VII, 1989, p. 5-24.

7. Larrieu-Duler M., Les puits funéraires de Lectoure (Gers), Mémoire de la Société Archéologique du Midi de la France, XXXVIII, 1973, p. 9-68.

8. Gallia, Informations archéologiques, XXII, 2, 1964, p. 451-452.

9. Les fouilles récentes de R. Boudet ont montré l'existence de nouveaux fours 

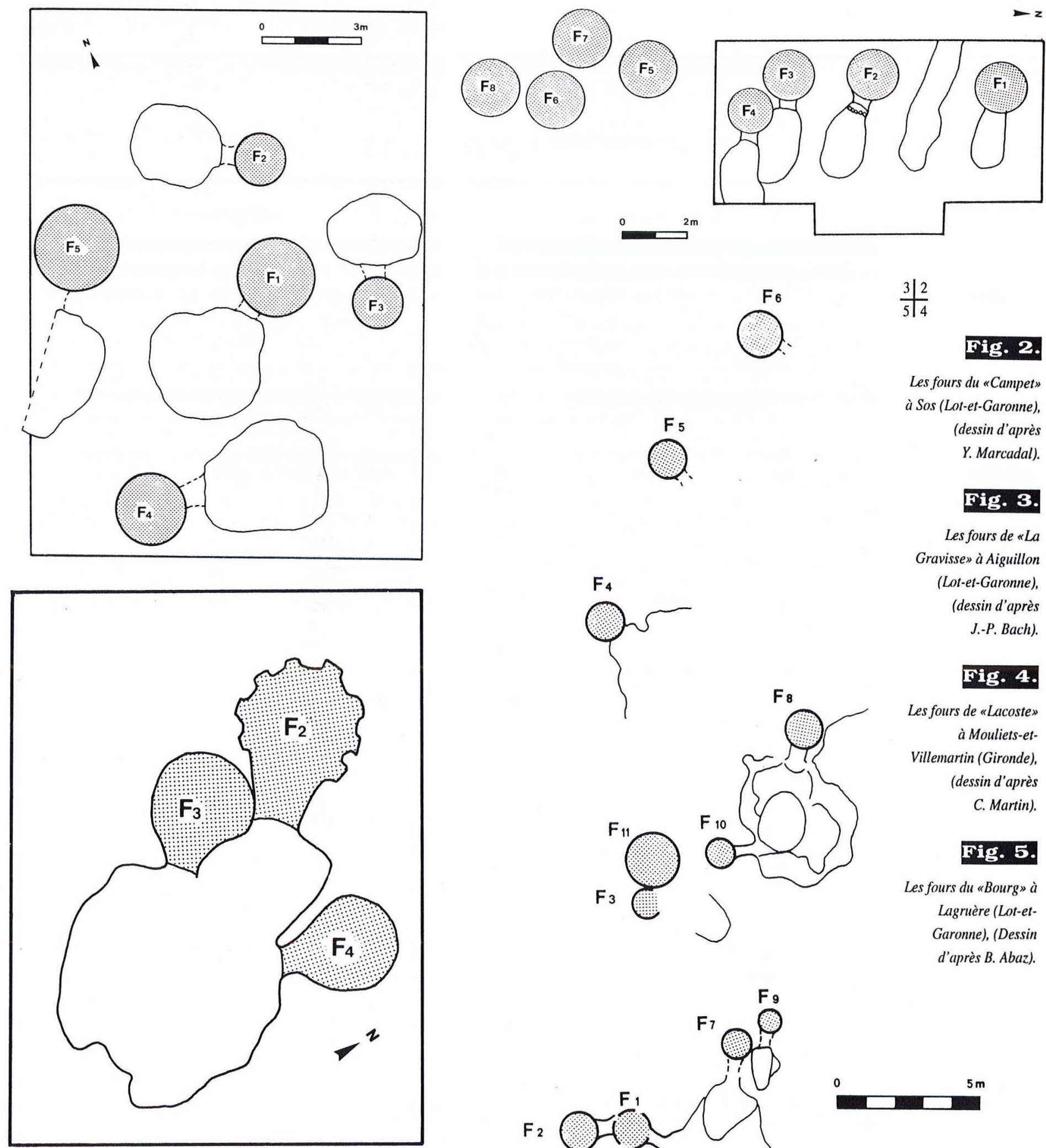

Les fours du «Campet» à Sos (Lot-et-Garonne), (dessin d'après Y. Marcadal).

Les fours de $\approx L a$ Gravisse» à Aiguillon (Lot-et-Garonne), (dessin d'après J.-P. Bach).

Fig. 4.

Les fours de «Lacoste» à Mouliets-etVillemartin (Gironde), (dessin d'après C. Martin).

Fig. 5.

Les fours du «Bourg» à Lagruère (Lot-etGaronne), (Dessin d'après B. Abaz).

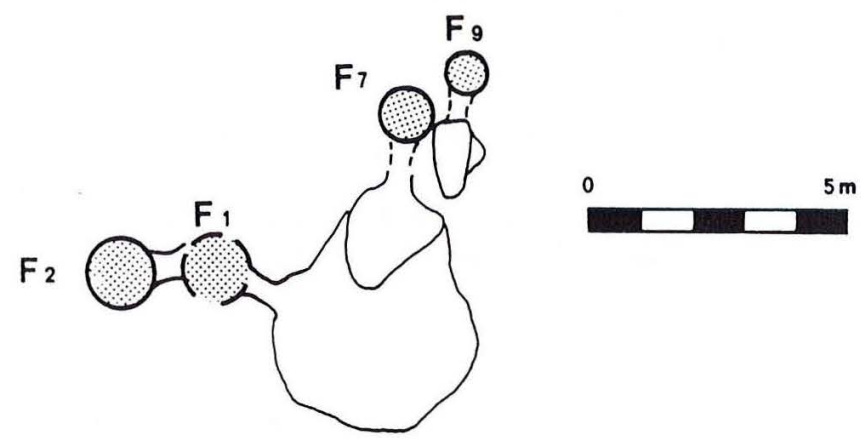




\section{Fig. 6.}

L'alandier effondré $d u$ four $n^{\circ} 7$ de «Lacoste» Mouliets-et-Villemartin (Gironde),

(Cliché S. Faravel).

\section{Fig. 7.}

Un alandier des fours de "La Gravisse» à Aiguillon (Lot-et-Garonne), (cliché A. Réginato).

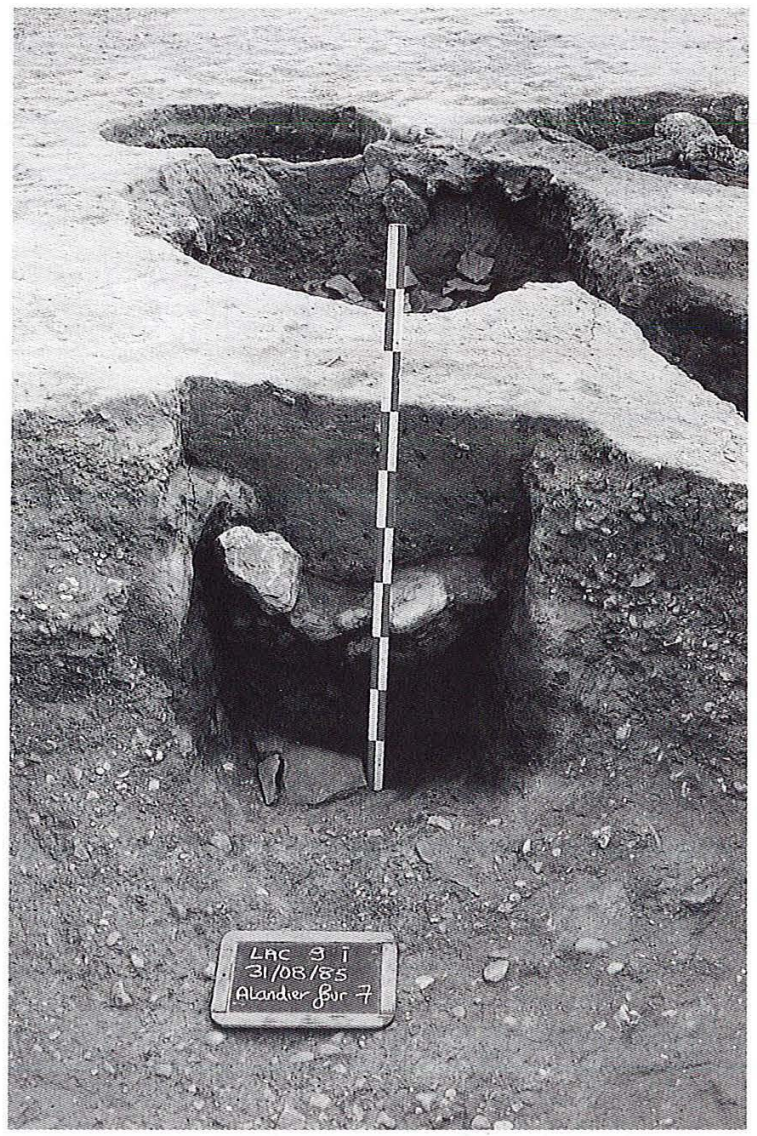

\section{Localisation des ateliers par rapport à l'habitat}

Les fours sont toujours regroupés, souvent à quelques mètres les uns des autres. Dans tous les cas, que cela soit à Agen, Mouliets-et-Villemartin, Aiguillon, Lagruère ou Sos, les chercheurs pensent qu'il existe de nombreux autres fours à proximité de ceux déjà explorés; certains ont déjà pu le vérifier (Lagruère, Sos, Mouliets-et-Villemartin et Aiguillon). Parfois il existe plusieurs secteurs de production sur le même site comme c'est le cas à Aiguillon, Lagruère et Sos, peutêtre aussi à Agen.

Les fours sont généralement isolés de l'habitat et sont regroupés en périphérie; ils peuvent parfois en être assez éloignés comme à Sos où ils se situent à 500 mètres environ de la zone d'habitat. Par contre à Aiguillon ils en sont très proches et recoupent même des niveaux d'une occupation peu antérieure à leur mise en place ${ }^{10}$.

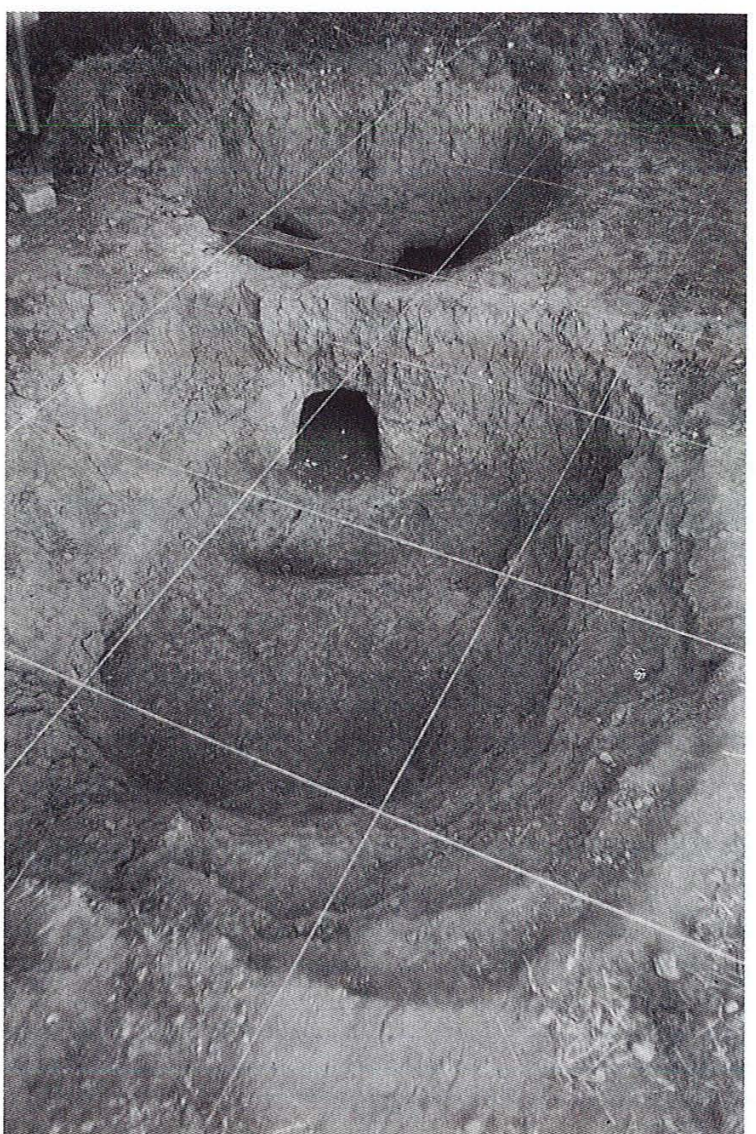

Les sites dont dépendent ces ateliers de production céramique sont soit des grands sites ouverts de plaine comme Mouliets-et-Villematin, Aiguillon et certainement Lagruère, ou des oppida (Agen et Sos). Il s'agit donc, dans tous les cas d'habitats groupés occupant des superficies de plusieurs hectares. Pour l'instant nous ne connaissons pas suffisamment les habitats isolés pour savoir si ces derniers étaient dotés de leurs propres structures de production.

La position des officines est en relation avec la situation de l'habitat et non pas avec celle des gisements de terres utilisées pour la céramique et pour la confection des fours. Dans plusieurs cas l'argile est très proche, par contre à Mouliets-et-Villemartin, les affleurements sont à plus d'un kilomètre. Il semble d'ailleurs que la distance qui sépare l'officine des zones de matières

10. Réginato A., Archéologie en Aquitaine, 5,.1986, p. 61-65. 
premières peut avoir une incidence sur certaines variations typologiques constatées entre certains fours, d'une officine à l'autre.

\section{Organisation interne des ateliers}

Les fours se présentent sous la forme de petites unités de production qui s'organisent suivant deux schémas différents:

1. La forme la plus simple est celle du four seul accompagné d'une petite fosse lui donnant accès. A Sos (fig. 2) ces petites unités sont disposées en parallèle, les unes à côté des autres, à Aiguillon (fig. 3), elles semblent être disposées un peu dans tous les sens, sans doute dans un souci d'économie d'espace car, ici, les fours, nous l'avons vu, sont très proches de la zone d'habitat.

2. La seconde forme d'organisation est la vaste fosse ronde ou oblongue qui donne accès, dans un premier temps, à un seul four, puis à un autre et quelquefois à un troisième. Ce type d'organisation est clairement lisible à Mouliets-et-Villemartin (fig. 4) ainsi qu’à Lagruère (fig. 5). Ces deux derniers sites possèdent également des fours organisés suivant le premier schéma décrit ci-dessus.

Ence qui concernel'orientation des fours, on constate plusieurs cas de figure : à Sos, tous les fours sont orientés à l'ouest, à Mouliets-et-Villemartin, entre l'ouest et le nord, à Lagruère, entre l'ouest et le sudouest. Si nous ne connaissions pas les fours d'Aiguillon, nous en conclurions qu'il existe une volonté de la part des potiers d'orienter leurs fours par rapport au sens des vents dominants de notre région, qui varient du nord au sud-ouest (pour améliorer le tirage et peut-être se protéger des fumées). Les fours d'Aiguillon forment ici un magnifique contre-exemple qui nous interdit de formuler certaines conclusions trop hâtives.

\section{Les fours}

Tous les fours recensés sont du type paracirculaire à alandier et à tirage vertical.

La longueur des alandiers varie de 0,5 à $0,7 \mathrm{~m}$, les soles ont un diamètre qui varie de 0,9 à 2 mètres.

Les alandiers sont soit bâtis (fig. 6), comme à Mouliets-et-Villemartin, soit directement taillés dans
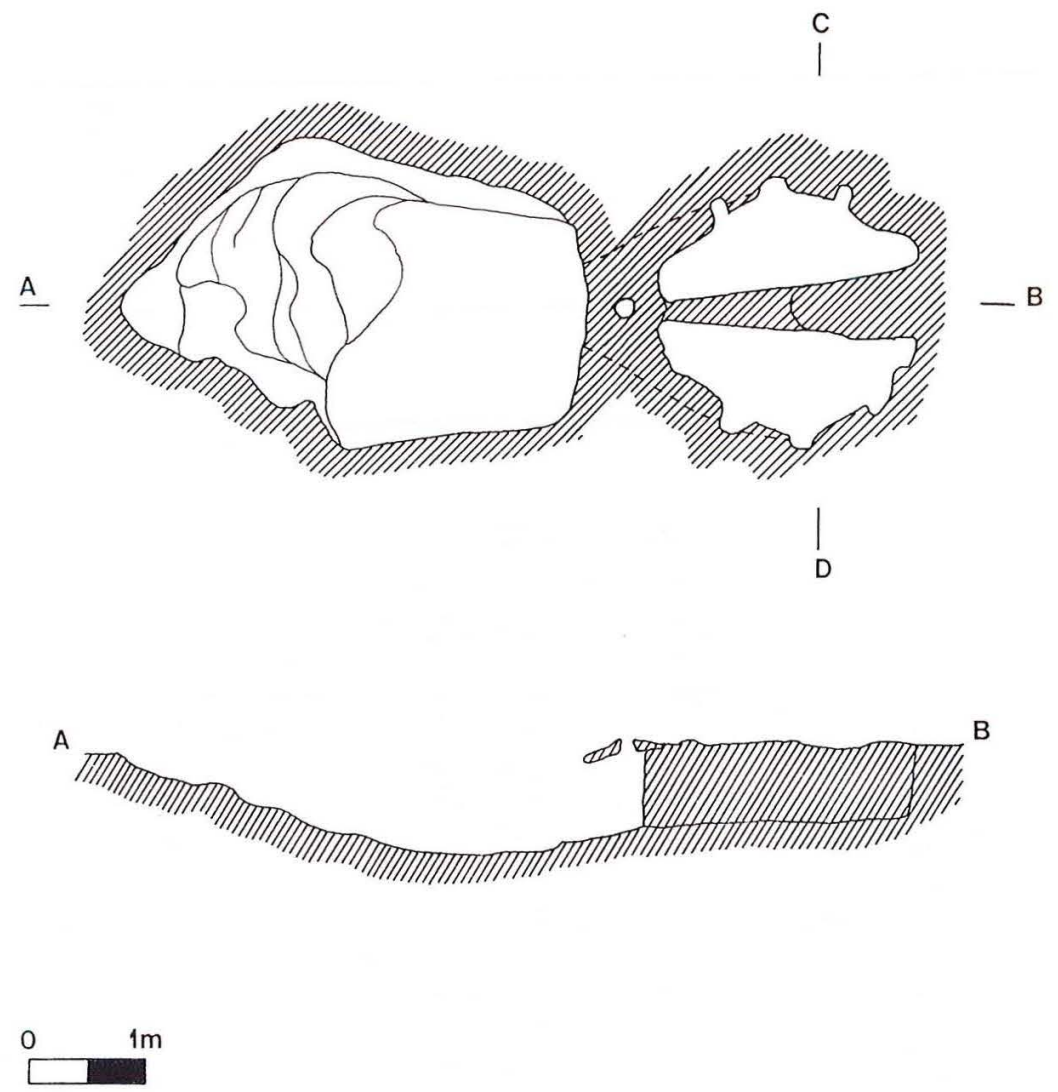

C

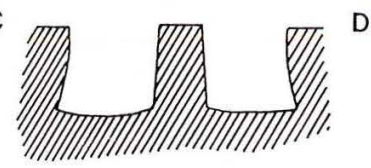

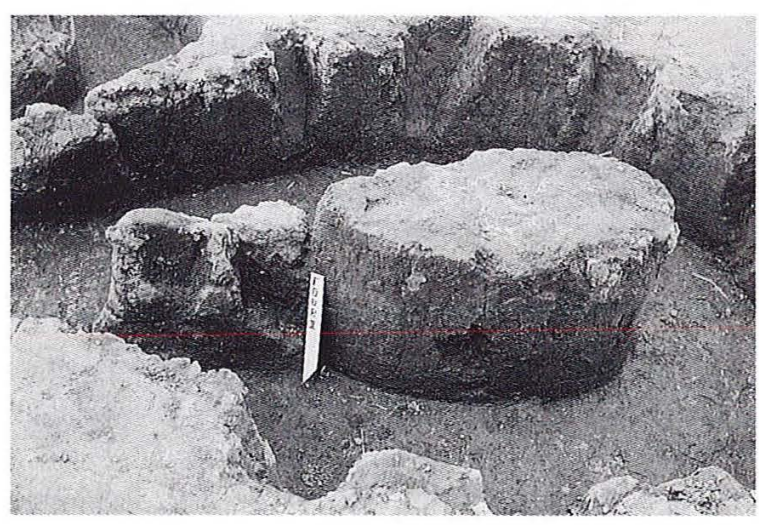

le substrat géologique. Dans le premier cas, ils sont de section rectangulaire, dans le second, ils sont de section trapézoïdale ou triangulaire, commeà Sos ouà Aiguillon (fig. 7). Les alandiers bâtis le sont avec des matériaux réfractaires : calcaire, grès ou molasse revêtu d'un enduit d'argile.
Fig. 8.

Le four $n^{\circ} 1 \mathrm{du}$ «Bourg» à Lagruère

(Lot-et-Garonne), (dessin d'après J.-P. Philhastre).

\section{Fig. 9.}

Un four à pilier central vertical de Lagruère (Lot-et-Garonne), (cliché B. Abaz). 

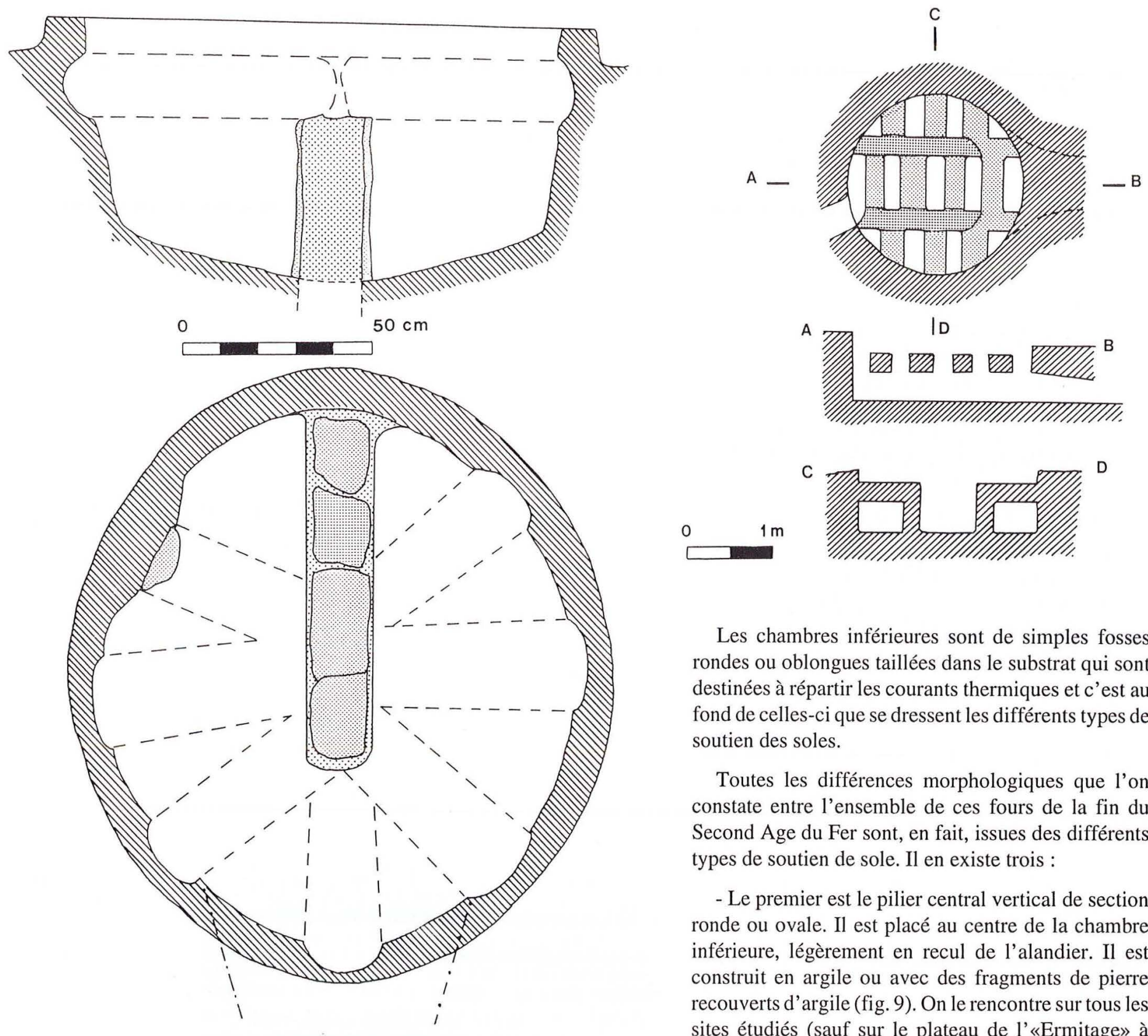

A
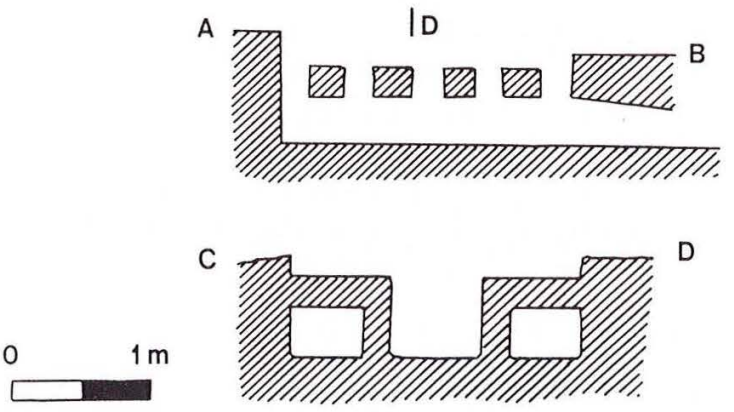

$11 \mid 10$

\section{Fig. 10.}

Le four $n^{\circ} 2$ du site de l'«Ermitage» à Agen

(Lot-et-Garonne), (dessin d'après A. Laurent).

\section{Fig. 11}

Le four $n^{\circ} 5$ de Sos (Lot-et-Garonne), (dessin d'après Y. Marcadal).
C'est àl'intérieur et surtout àl'entrée de ces alandiers que le foyer d'alimentation du four était entretenu. Dans la majorité des cas le fond de l'alandier se trouve en léger contrebas par rapport au fond de la chambre inférieure du four, afin d'améliorer le tirage.

L'alandier du four $\mathrm{N}^{\circ} 1$ de Lagruère possède une très singulière perforation de sa voûte (fig. 8). Il doit s'agir, d'après l'inventeur, d'un système permettant d'intervenir sur la température de cuisson ou sur son atmosphère.
Les chambres inférieures sont de simples fosses rondes ou oblongues taillées dans le substrat qui sont destinées à répartir les courants thermiques et c'est au fond de celles-ci que se dressent les différents types de soutien des soles.

Toutes les différences morphologiques que l'on constate entre l'ensemble de ces fours de la fin du Second Age du Fer sont, en fait, issues des différents types de soutien de sole. Il en existe trois :

- Le premier est le pilier central vertical de section ronde ou ovale. Il est placé au centre de la chambre inférieure, légèrement en recul de l'alandier. Il est construit en argile ou avec des fragments de pierre recouverts d'argile (fig. 9). On le rencontre sur tous les sites étudiés (sauf sur le plateau de l'«Ermitage» à Agen).

- Le second type est le muret de refend qui partage la chambre inférieure en deux parties. Sur le site de l'«Ermitage», il existe un système à double mur de refend (fig. 10) qui doit permettre d'augmenter la surface de la sole puisque cette dernière est très grande (2 mètres). On rencontre également des murs de refend à Lagruère (fig. 8) et à Aiguillon. Le type de soutien de sole à mur de refend est presque aussi répandu que celui à pilier central. 


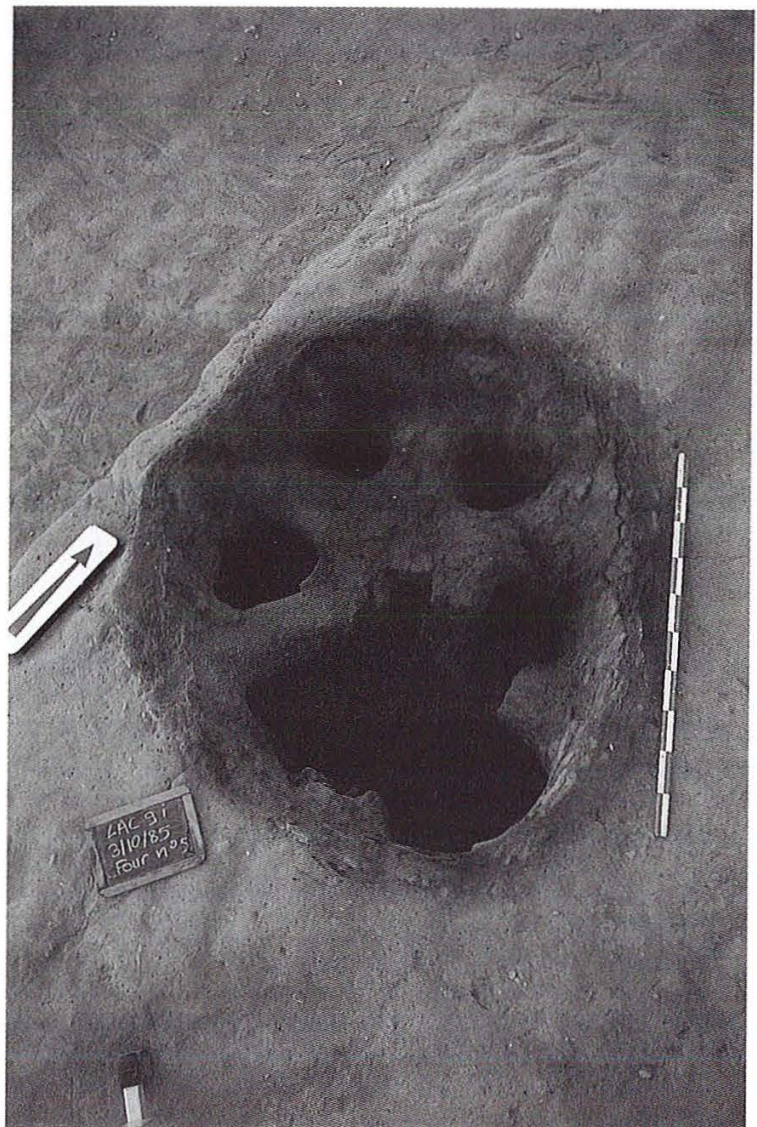

- Le troisième et dernier type de soutien de sole est la languette, c'est à dire un mur de refend écourté du coté de l'alandier du four. Ce système est présent à Sos (fig. 11) et à Aiguillon mais demeure moins fréquent que les deux précédents.

Il ne semble pas exister de rapport typochronologique entre ces différents systèmes de soutien de sole; à Aiguillon, on rencontre les trois types de manière quasiment simultanée (fig. 12). Par contre, à Moulietset-Villemartin, seul le système à pilier central vertical est représenté. Cette singularité est vraisemblablement due à l'éloignement des matières premières par rapport aux fours. La confection d'un mur de refend ou d'une languette nécessite beaucoup plus d'argile qu'un pilier central. D'ailleurs, le diamètre moyen des fours de Mouliets-et-Villemartin s'en ressent, puisqu'il est largement inférieur à celui des autres officines.

Les soles sont généralement rayonnantes, confectionnées à partir de luts horizontaux qui prennent naissance à la base des parois du laboratoire et s'appuient sur le pilier central, le mur de refend ou la «languette».
1: pilier central cylindrique

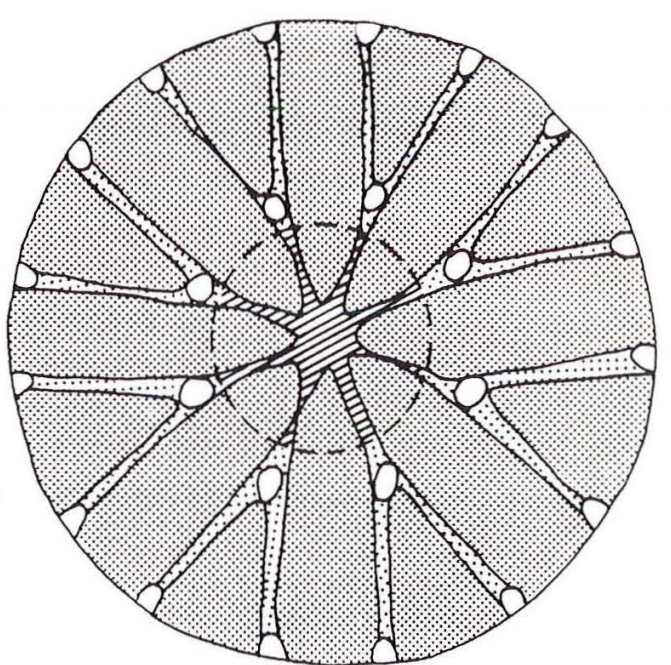

$2:$ mur de refend

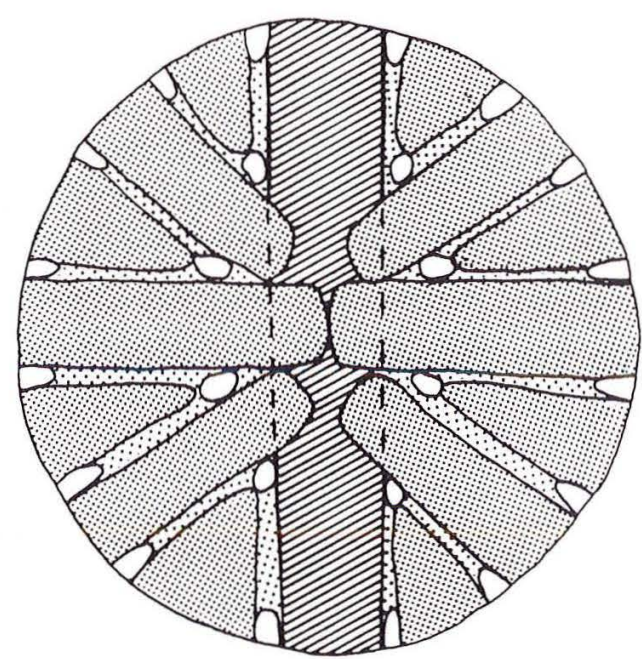

$3:$ languette

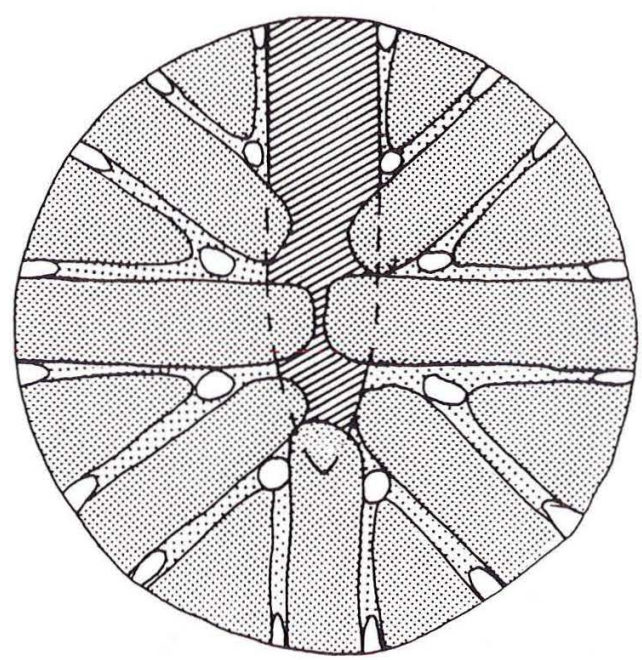

$13 \mid 12$
Fig. 12 .

Les trois types de soutien de sole de "La Gravisse» à Aiguillon (Lot-et-Garonne), (dessin d'après A. Réginato).

Fig. 13. 

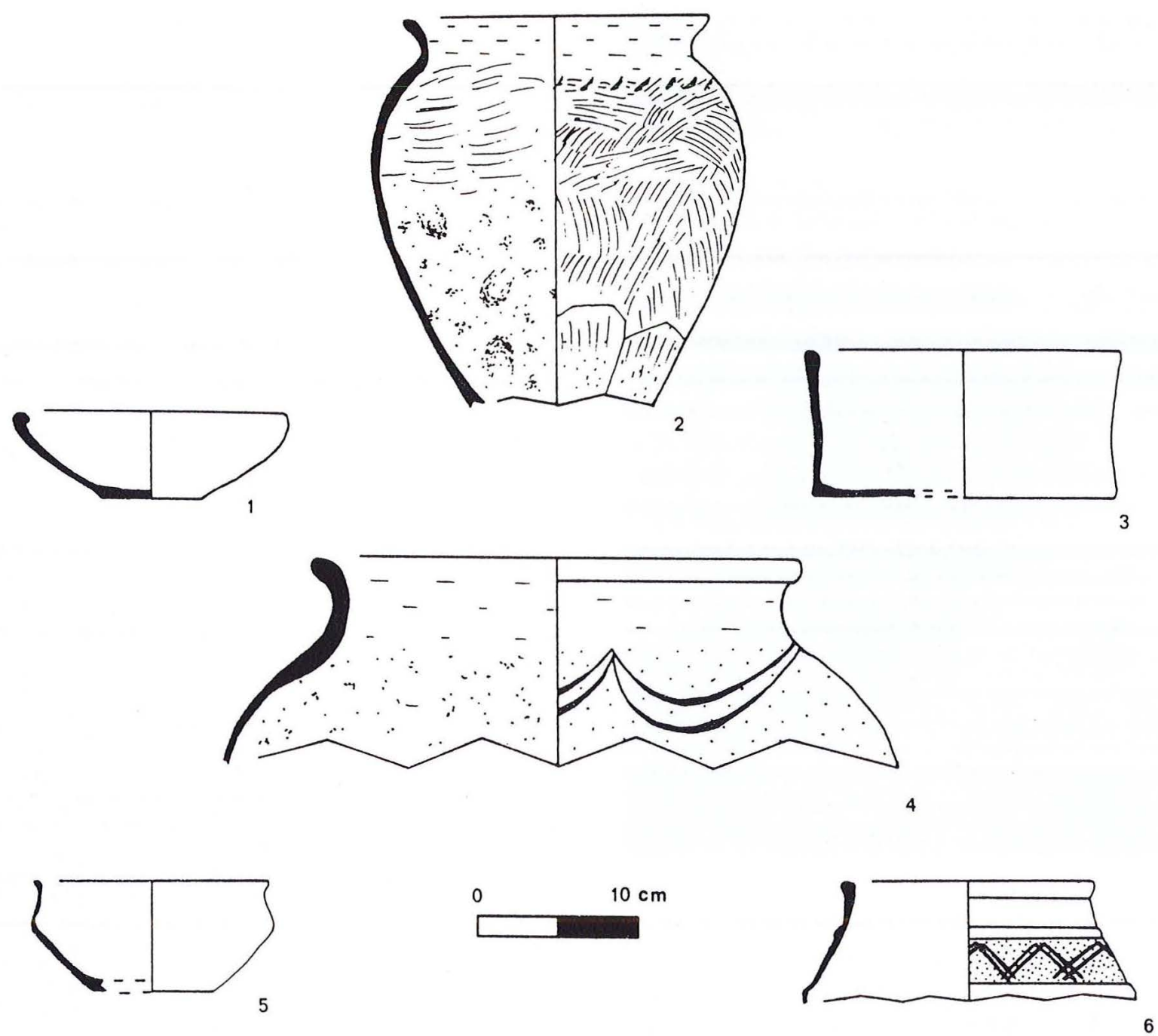

\section{Fig. 14.}

La production des fours de "Lacoste» à

Mouliets-et-Villemartin

(Gironde),

1 à 4 : formes

courantes,

5 à 8 : formes rares,

(dessin Ch. Sireix).
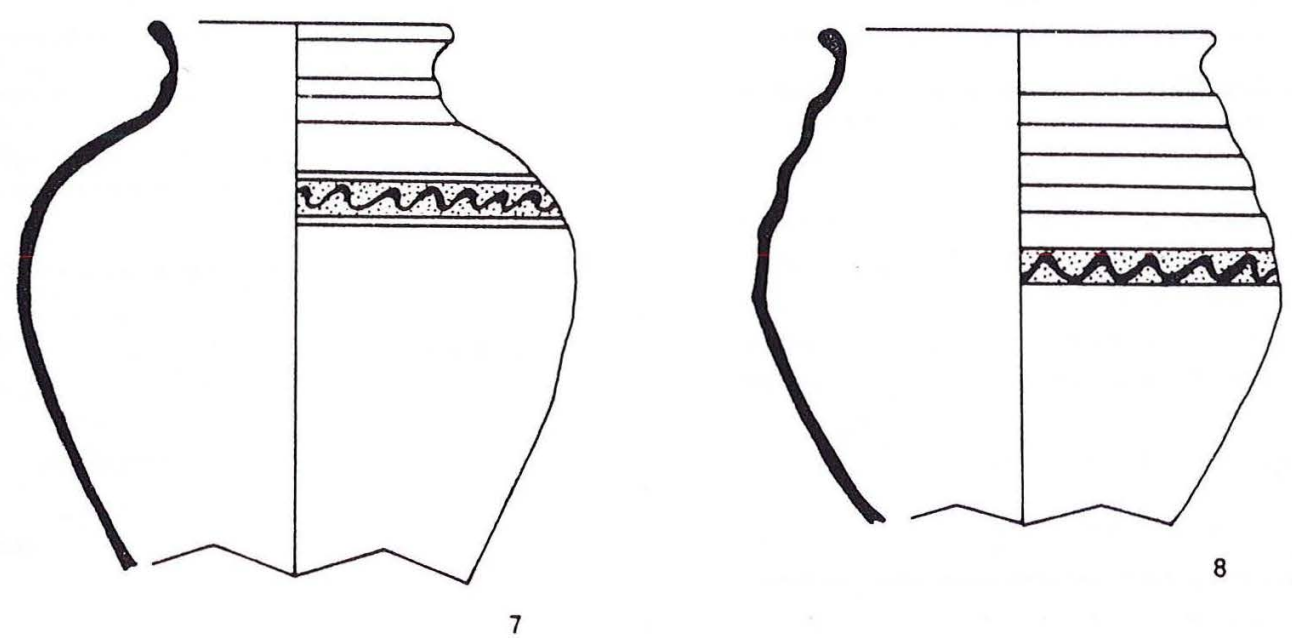

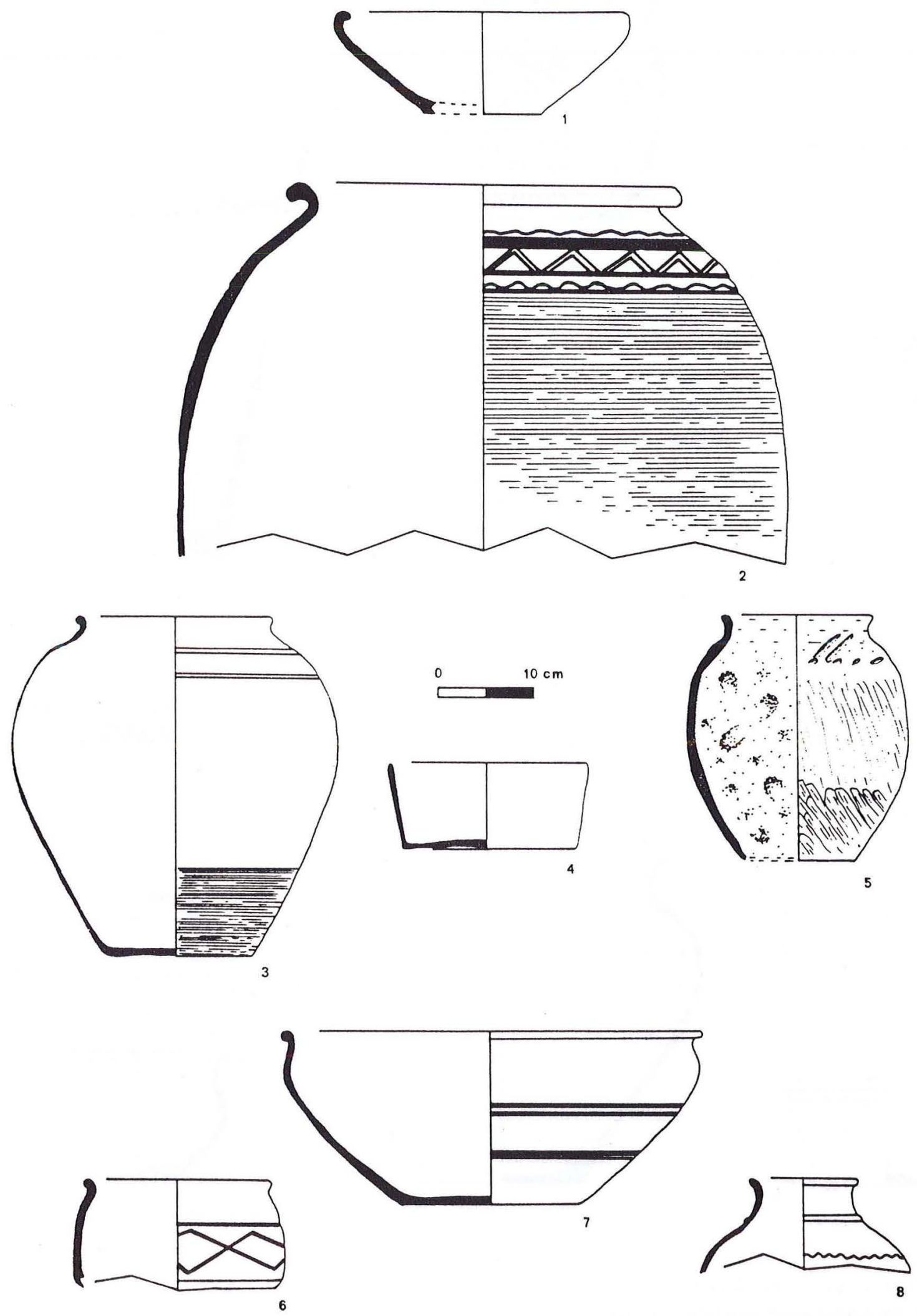

\section{Fig. 15.}

La production des fours de «La Gravisse» à Aiguillon (Lot-et-Garonne), Ià 5 : formes courantes, 6 à 8 : formes peu fréquentes, (dessin d'après A. Réginato). 

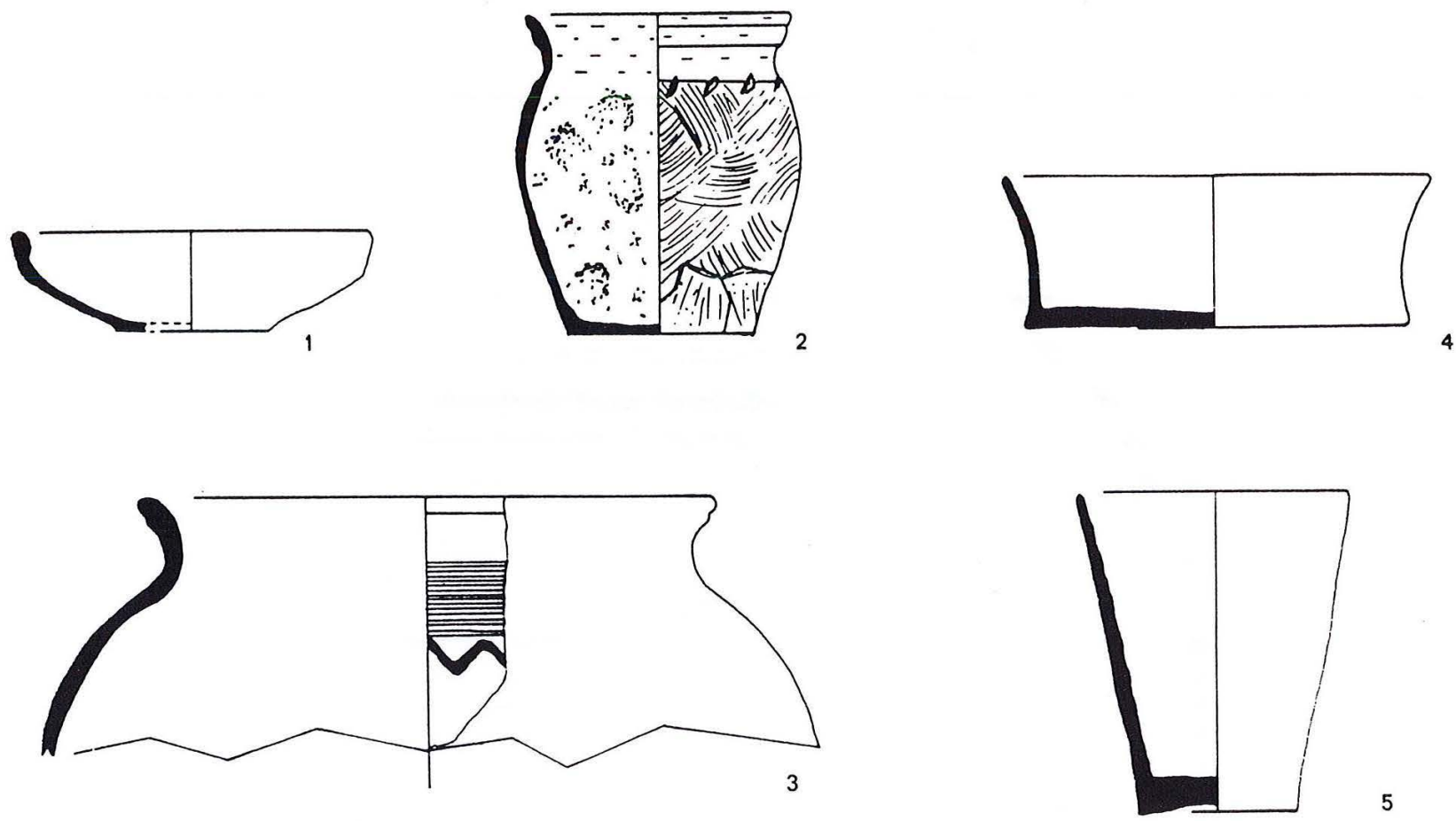

0 $10 \mathrm{~cm}$
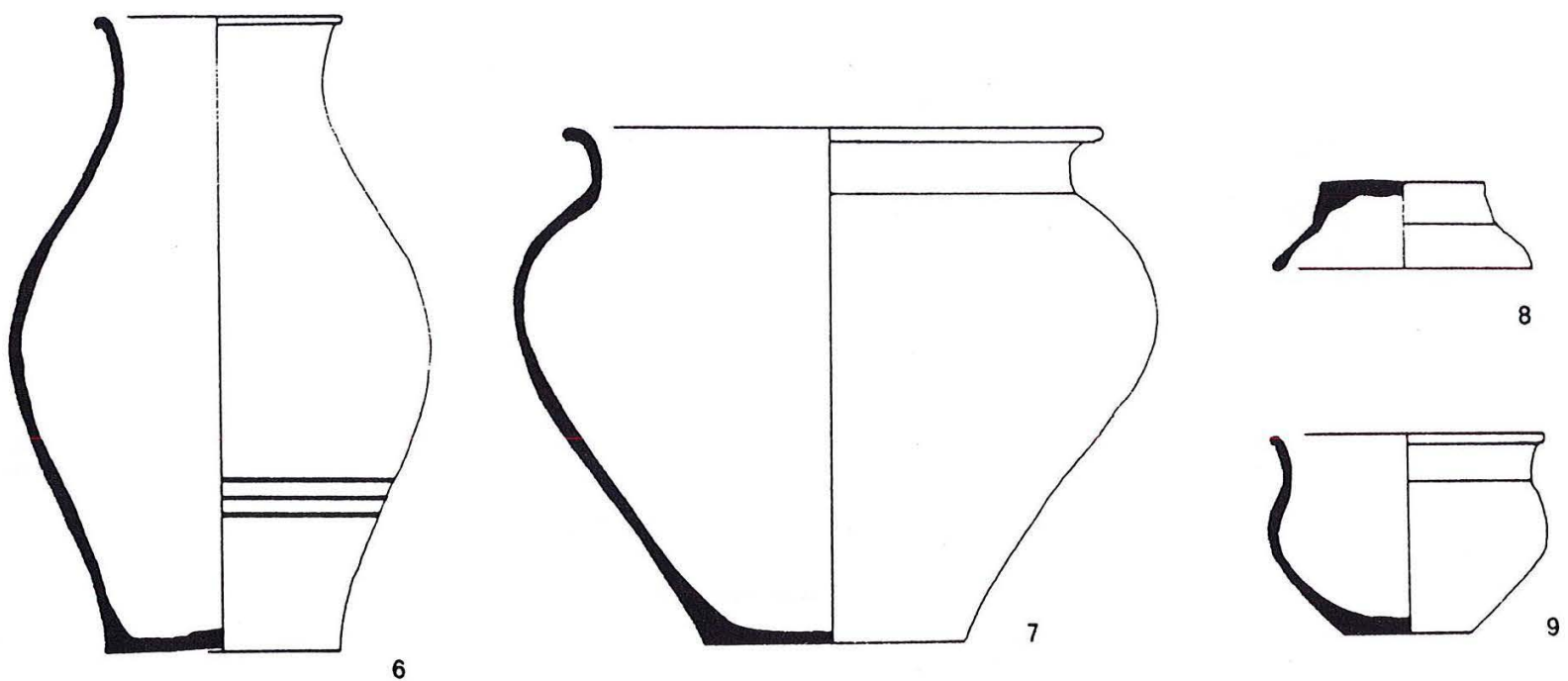

\section{Fig. 16.}

La production des fours $d u$ «Bourg» à Lagruère (Lot-et-Garonne),

1 à 5 : formes courantes, 6 à 9 : formes peu fréquentes, (dessin d'après $P$. Négro). 

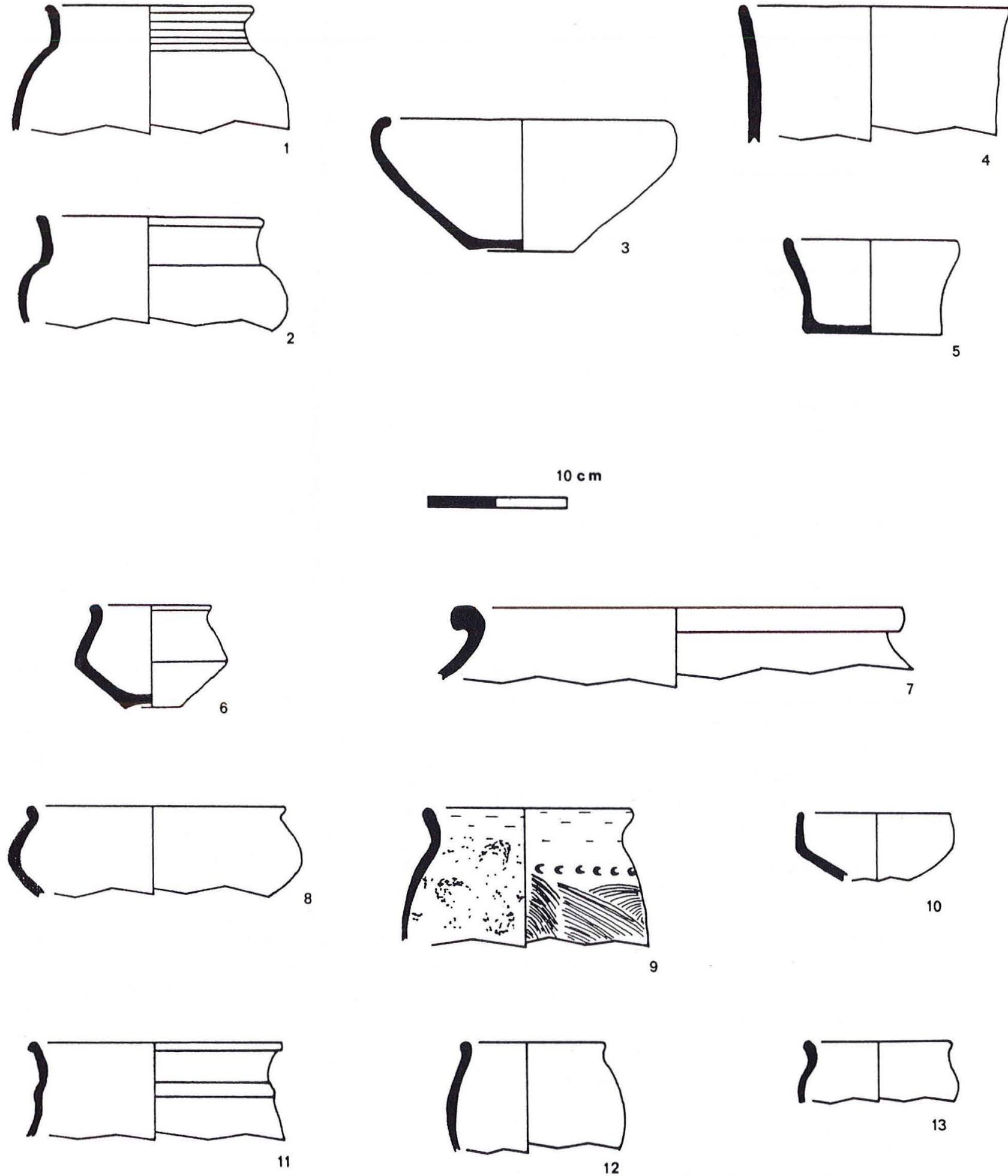

13 


\begin{tabular}{|lr|}
\hline TYPE DE VASE & \multicolumn{1}{c|}{} \\
\hline Terrine & 37,64 \\
\hline Urne peignée & 29,84 \\
\hline Dolium & 9,89 \\
\hline Gobelet & 5,11 \\
\hline Urne biconique & 1,59 \\
\hline Jatte carénée & 0,56 \\
\hline Urne globulaire & 0,50 \\
\hline Autre & 10,43 \\
\hline Indéterminé & 4,44 \\
\hline
\end{tabular}

Mouliets-et-Villemartin, «Lacoste»

\begin{tabular}{|lr|}
\hline TYPE DE VASE & \multicolumn{1}{c|}{} \\
\hline Terrine & 31,72 \\
\hline Urne peignée & 8,97 \\
\hline Dolium & 21,08 \\
\hline Urne ovoïde & 12,75 \\
\hline Gobelet & 7,25 \\
\hline Jatte carénée & 2,06 \\
\hline Urne biconique & 1,17 \\
\hline Autre & 4,56 \\
\hline Indéterminé & 10,44 \\
\hline
\end{tabular}

Aiguillon, «La Gravisse»

A Aiguillon, un système de rayons intermédiaires permet d'obtenir une sole avec de nombreux petits orifices qui se rapprochent typologiquement de carneaux (fig. 12). A Mouliets-et-Villemartin, ces espaces en forme d'amande sont si grands qu'ils ont souvent laissé passer des rebuts de cuisson à l'intérieur de la chambre inférieure (fig. 13).

Aucun laboratoire de cuisson n'a bien évidemment conservé sa couverture. La question de la présence de voûte reste souvent sans réponse. Il semble qu'il faille à tout prix rejeter l'idée d'une voûte détruite à chaque cuisson et plutôt imaginer un système ressemblant plus à une cheminée ou un cône, qu'à une coupole. Un système à obturation horizontale est, en effet, plus facile à réaliser et permet un meilleur accès au laboratoire de cuisson. Ce type de construction peut ainsi expliquer la présence des très nombreux fragments d'amphores surcuites que l'on trouve souvent parmi les rebuts de cuissons (ces fragments d'amphores étant directement posés sur le sommet de l'empilement des vases à cuire).

Le site d'Aiguillon est le seul à avoir fourni de nombreux fragments d'argile cuite portant des traces d'armatures ou de branchage. Les autres sites four-

\begin{tabular}{|lr|}
\hline TYPE DE VASE & \multicolumn{1}{c|}{$\boldsymbol{~}$} \\
\hline Terrine & 48,05 \\
\hline Urne peignée & 20,16 \\
\hline Dolium et urne ovoïde & 13,13 \\
\hline Gobelet & 6,04 \\
\hline Urne biconique & 1,04 \\
\hline Jatte carénée & 0,59 \\
\hline Autre & 3,77 \\
\hline Indéterminé & 7,22 \\
\hline
\end{tabular}

Lagruère, «Le Bourg»

\begin{tabular}{|lr|}
\hline TYPE DE VASE $\quad$ \% \\
\hline Urne ovoïde et glob. & 45,61 \\
\hline Terrine & 30,02 \\
\hline Gobelet & 13,51 \\
\hline Petit vase biconique & 2,19 \\
\hline Dolium & 1,85 \\
\hline Urne peignée & 1,27 \\
\hline Jatte carénée & 1,27 \\
\hline Autre & 2,78 \\
\hline Indéterminé & 1,50 \\
\hline
\end{tabular}

Sos, «Le Campet»

nissent souvent des fragments de parois avec des traces de lissage à la main sur la partie interne, concave.

Les cuissons sont, dans la majorité des cas oxydantes avec une post-cuisson réductrice parfois irrégulière. La nature des rebuts de cuisson peut nous renseigner sur les capacités thermiques des fours de chaque officine. Ils peuvent être déformés voire boursouflés, comme dans les tessonières d'Aiguillon et dans une fosse d'accès à un four non fouillé de Lagruère. Il n'en existe pas de ce type à Mouliets-et-Villemartin où, par contre, on trouve fréquemment des vases sous-cuits à pâte tendre et très friable. Les fours de Mouliets-et-Villemartin ne semblent pas avoir atteint les mêmes températures de cuisson que ceux de Lagruère ou d'Aiguillon. Il existe également de nombreux éclats «cupulaires» dus à des chocs thermiques pendant les cuissons ou à des tessons recuits dans les alandiers et les chambres inférieures.

La durée d'utilisation des fours est inconnue, mais on constate très souvent des traces de réfections de parois et même parfois des réparations qui traduisent la volontédes potiers d'utiliser au maximum leur structure de chauffe. 


\section{Ta production}

La production n'est pas très variée, les formes les plus fréquentes étant les terrines (ou ecuelles), les urnes peignées, les dolia et les gobelets (fig. 14 à 17). Seuls les fours de Sos offrent un éventail de formes différentes; ces fours nous semblent être les plus récents parmi ceux considérés dans cette étude ${ }^{11}$. Nous ne connaissons pas la production des fours d'Agen.

Quatre formes différentes se partagent la majorité de la production de trois principales officines ${ }^{12}$ (fig. 18). Ces quatre formes apparaissent parfois dans des proportions quelque peu différentes, et le cas d'Aiguillon est très intéressant avec une forte proportion de dolium et d'urnes ovoïdes (qui sont les mêmes formes dans un gabarit inférieur).

Le site de «La Gravisse» à Aiguillon est le seul à avoir livré, parmi les rebuts de cuisson de ses fours, des fragments de dolia possédant des marques imprimées sur l'épaulement du vase avant cuisson. Ces marques ne sont présentes que sur les dolia (fig. 19), elles sont simples, doubles ou triples ${ }^{13}$, nous ignorons si elles ont un rapport avec la contenance des vases.

Ces dolia sont produits en forte proportion par rapport aux autres sites et très certainement exportés. Ils peuvent nous renseigner sur l'une des principales activités économiques du site d'Aiguillon, mais le problème est de savoir si l'on a exporté des vases avec leur contenu (vraisemblablement des céréales) ou simplement des vases vides destinés à recevoir un contenu surun autre site. A Sos, un fragment de dolium estampillé découvert dans un sondage en $1989^{14}$, a de fortes chances de provenir du centre potier d'Aiguillon (fig. 20).

Il est impossible de déterminer l'ampleur de la diffusion des produits des autres officines. Il semble que celle-ci soit avant tout destinée à la propre consommation des habitats dont elles dépendent et certainement à leurs satellites.

Aucun ateliers de préparation des terres et de montage des vases n'a été localisé. Les seuls indices archéologiques se résument à quelques brunissoirs découverts près des fours d'Aiguillon et dans une zone d'habitat de Mouliets-et-Villemartin.

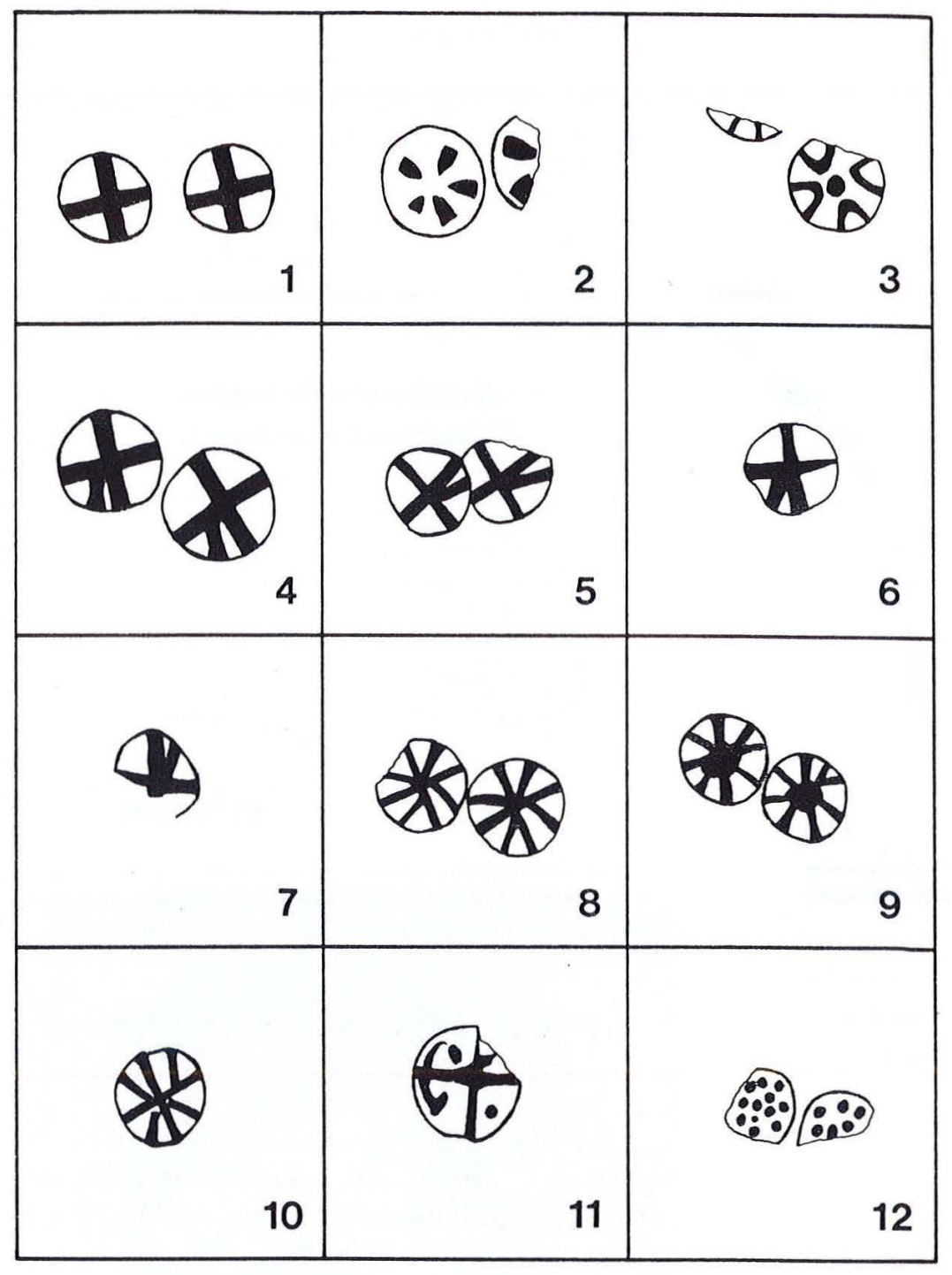

0

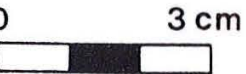

Fig. 19.

11. La partie sommitale du remplissage de ces fours a fourni de nombreux fragments d'amphores de type Dressel IB associés à des fragments d'amphores de type Pascual 1 et Dressel 7/11.Leur période de fonctionnement ne peut donc être antérieure à la Conquête romaine et doit vraisemblablement se situer dans le courant de la seconde moitié du Ier siècle avant J.-C..

12. Ces pourcentages sont obtenus à partir du comptage systématique de la totalité des bords et des lèvres appartenant à des vases issus uniquement des fours et des tessonières. Ils ne sont pas le reflet direct de la production de chaque officine, car aucun facteur de pondération n'a été introduit, mais ils permettent de comparer efficacement les productions des différentes officines.

13. Découverte de dernière minute, information : A. Reginato que nous remerçions vivement.

14. Lambert P. , Un sondage archéologique sur l'oppidum de Sos (Lot-etGaronne), Actes de la XIlème journée des archéologues gersois, 1990, p. $21-40$.
Les différents types dolia de "La Gravisse»

à Aiguillon

(Lot-et-Garonne), (dessin A. Réginato et Ch. Sireix). d'estampilles sur les 


\section{Origine et pérennité}

Les deux fours de potiers du Second Age du Fer les plus anciens connus dans notre région, ont été découverts sur le site de hauteur des «Grands-Vignes» à Sainte-
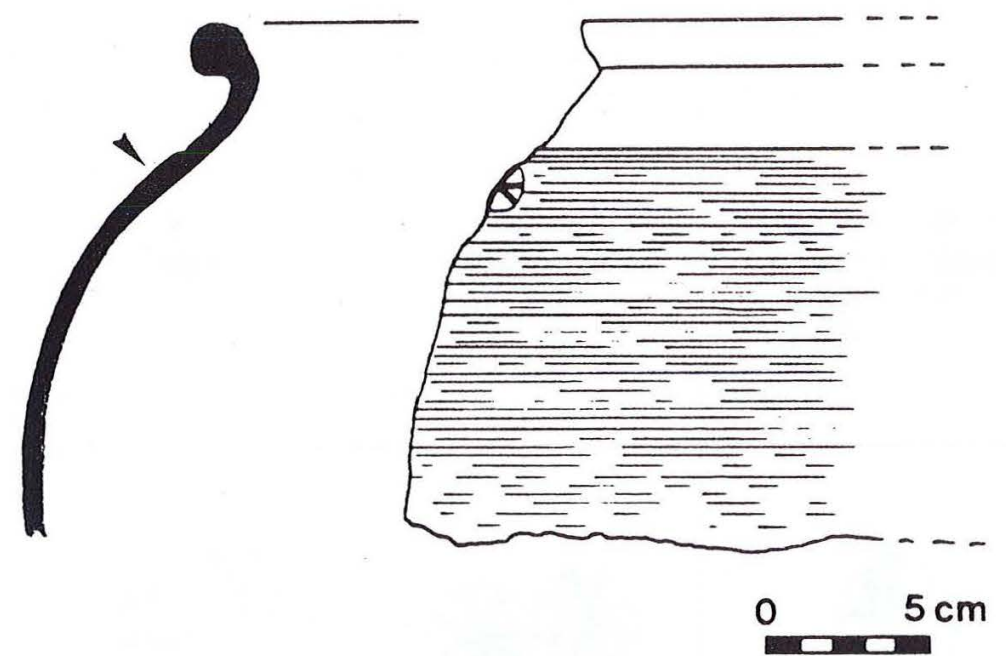

\section{Fig. 20.}

Dolium estampillé de Sos (Lot-et-Garonne), (dessin d'après P. Lambert).

\section{Fig. 21a, b.}

Le four $n^{\circ} I$ des "Grands-Vignes II" à Sainte-Florence (Gironde), (cliché Ch. Sireix).

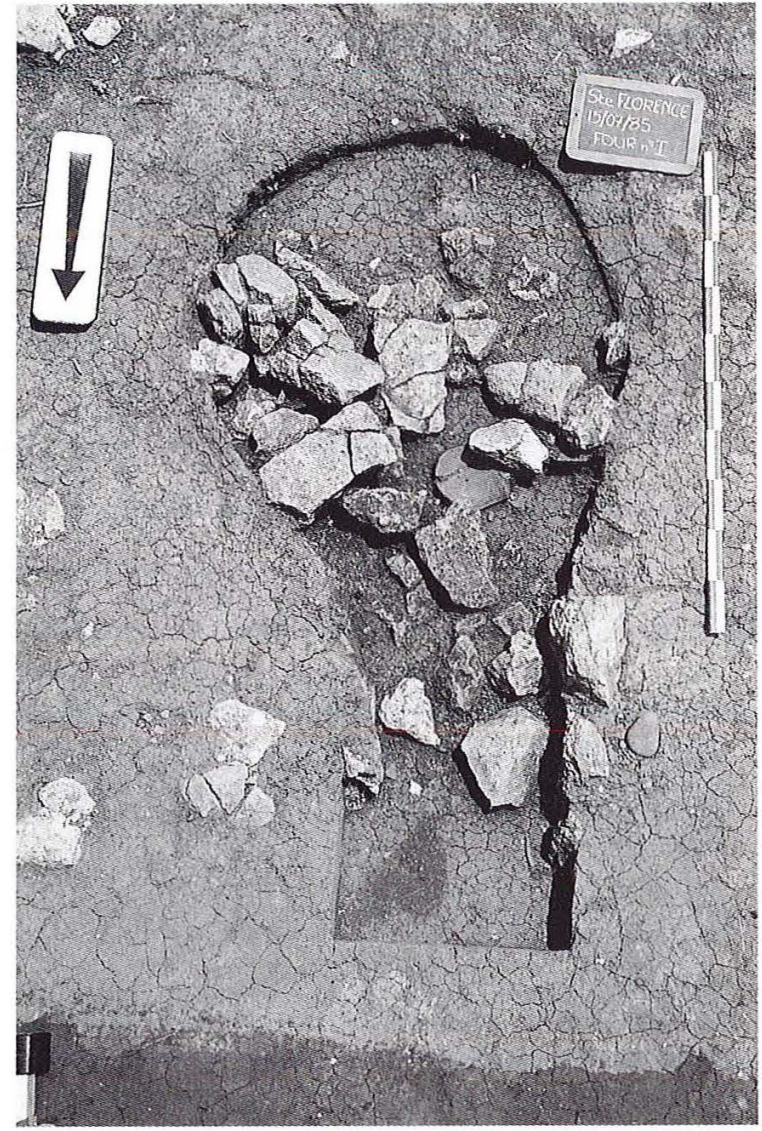

Florence ${ }^{15}$ et date de la seconde moitié du IVe siècle avant notre ère (fig. 21). Un seul de ces deux fours est bien conservé, il s'agit d'un four paracirculaire à alandier et à sole rayonnante soutenue par un pilier central vertical. Ce four possède une chambre inférieure qui est peu profonde. Sa production est typiquement laténienne: vases carénés, vases à décors de baguettes et à couverte rouge hématite. Certaines formes sont montées à l'aide d'un tour.

Ce four très ancien peut donc être rapproché typologiquement de ceux qui sont construits deux à trois siècles plus tard dans la même région. Peut-être s'agitil ici d'un apport culturel dû à la phase d'expansion laténienne dans le sud-ouest de l'Europe que l'on a coutume de placer dans le courant du IVe siècle avant J.-C. Toujours est-il que les fours à sole rayonnante du

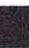

15. Pour plus de détail sur ce four et sur ce site voir : Sireix Ch., Le site protohistorique des Grands-Vignes II à Sainte-Florence (Gironde), Aquitania, VII, 1989, p. 5-24.

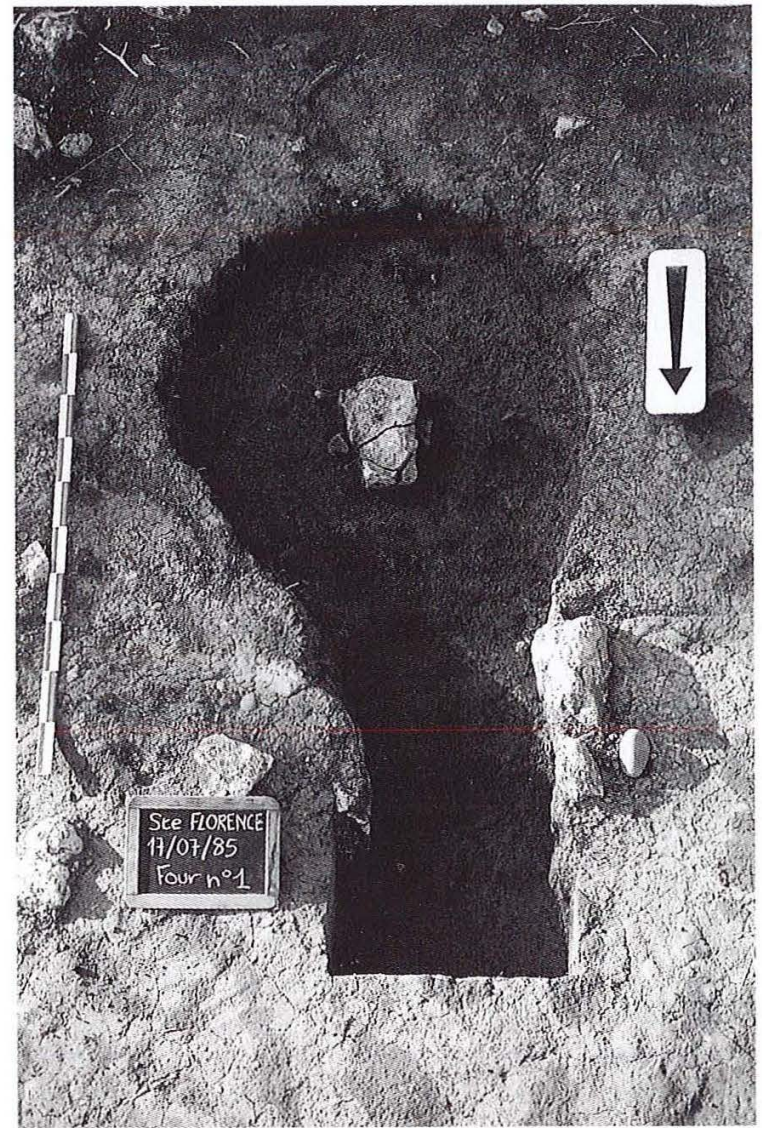


second age du Fer n'ont pas subi l'influence méditerranéenne qui a privilégier, dans le sud-est de la Gaule et durant les cinq siècles précédants notre ère, le type de fours à sole perforée de carneaux ${ }^{16}$.

Pour le début de la période gallo-romaine, une découverte très récente sur le site du Château à Vayres ${ }^{17}$ en Gironde, permet de constater la perduration des fours à mur de refend destinés à supporter des rayons (fig. 22). Datée du période comprise entre 20 et 1 avant J.-C., la production de ce four est composée de formes caractéristiques de la phase finale de Second Age du Fer associées aux nouvelles formes attribuables à la culture gallo-romaine. Des fours du même type, datables de règne d'Auguste, ont déjà été rencontrés à Saintes ${ }^{18}$ en 1970, et confirment donc le maintien d'une tradition «indigène» dans la conception des fours de potiers dans le nord de l'Aquitaine, au début de l'époque galloromaine.

Nous ignorons encore la période durant laquelle se fait le passage des soles à rayons à celles perforées de carneaux. Le site de Vayres doit pouvoir nous aider à répondre à cette question car ce dernier est dorénavant identifié comme étant un très grand centre de production de céramique gallo-romaine ayant diffusé ses produits sur un vaste territoire (jusqu'à 100 kilomètres) et ce, pendant au moins trois siècles.

La majorité des sites d'habitat groupé du Second Age du Fer connus en Aquitaine a livré, par des découvertes fortuites ou des fouilles systématiques, un nombre important de fours de potiers (au moins 35 dans les limites du cadre de cette étude). Leur zone de répartition se situe principalement le long de l'axe

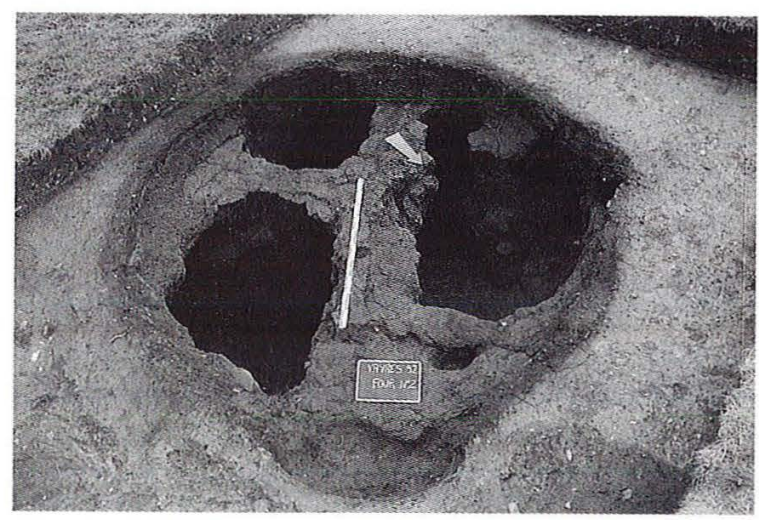

\section{Fig. 22.}

Le four augustéen du site du "Château» à Vayres (Gironde), (cliché Ch. Sireix) garonnais (ou dans sa proche périphérie) et dans la vallée de la Dordogne. Une telle concentration de fours, appartenant tous à un même type et à une même période, est exceptionnelle et demeure unique en Europe. Cette concentration bien ordonnée dans une zone géographique cohérente correspond à une entité culturelle homogène dont l'origine ne semble pas avoir été soumise à une à l'influence du monde méditerranéen.

16. Voir par exemple : Ugolini D., Olive M., Un four de potier du Ve siècle avant J.-C. à Béziers, place de la Madeleine, Gallia, 45, 1988, p. 13-28. et Py M., L'oppidum des Castels à Nages (Gard), Gallia, Suppl. XXXV, 1978, p. 121-123 et p. 321-323.

17. Sondage Ch. Sireix, effectué en mai 1992.

18. Gallia, Informations archéologiques, 33, 2, 1975, p. 374-375. Santrot M.-H. et J., Céramiques gallo-romaines d'Aquitaine, Centre de Recherche d'archéologie analytique, E.R.A. 584, Editions du C.N.R.S., Paris, 1979, p. 15-17. 